\title{
PROPIEDADES PSICOMÉTRICAS DEL INVENTARIO DE DEPRESIÓN ESTADO-RASGO (IDER) EN ADULTOS DE LIMA
}

\author{
Psychometric Properties of the State-Trait Depression \\ Inventory (ST-DEP) in a Adults of Lima
}

\author{
Lidia Sotelo L.*; Noemí Sotelo L.**; Sergio Dominguez L.***; \\ Ivonne Poma B.****; Emilio Cueto $Q . * * * * * ;$ David Alarcón $S . * * * * * *$; \\ Miguel Barboza P.*******; Oscar Padilla T.*********
}

\begin{abstract}
Resumen
En este estudio se analizaron las propiedades psicométricas de Inventario de Depresión EstadoRasgo (IDER) en una muestra de adultos de Lima Metropolitana, y se buscó obtener evidencias de validez que apoyen su uso tanto en población consultante y no consultante. Se hallaron indicadores de confiabilidad aceptables a través del método de consistencia interna (Alfa de Cronbach), así como evidencia de validez de contenido, validez factorial y de validez criterial. Concluyéndose que el IDER es un instrumento válido y confiable para evaluar el componente afectivo de la depresión.
\end{abstract}

Palabras claves: depresión, psicometría, confiabilidad, validez

\begin{abstract}
In this study the psychometric properties of State-Trait Depression Inventory (STDI) were analyzed in a sample of adults from metropolitan Lima, to obtain validity evidences that support its use both in the consulting and non-consulting population. Acceptable indicators of reliability were found through a method of internal consistency (Cronbach's alpha), as well as content validity evidence, factorial validity and of criteria validity. Therefore, the STDI is a valid and reliable instrument to evaluate the affective component of depression.
\end{abstract}

Keywords: depression, psychometric, reliability, validity

\footnotetext{
* Psicóloga. Docente de la Facultad de Psicología de la UNMSM y UIGV. lidia_sotelo@ hotmail.com ** Psicóloga. Docente de la Facultad de Psicología de la UNMSM y Trabajo Social UIGV.

*** Psicólogo. Docente de la Facultad de Psicología y Trabajo Social, UIGV.

**** Psicóloga. Docente de la Institución Educativa Saco Oliveros.

****** Psicólogo. Integrante de Humanitae SAC.

******* Bachiller en Psicología. UNMSM.

******* Estudiante de Psicología, UNMSM.

********* Bachiller en Psicología. UNMSM.
} 


\section{INTRODUCCIÓN}

Dentro de la psicología clínica y no siendo ajeno a otras aplicaciones del conocimiento psicológico, es frecuente que los trastornos depresivos sean uno de los problemas que continuamente han afectado a la población, y que surjan durante el padecimiento de otro trastorno y/o enfermedad. Es así que Sogi (1997, en Ministerio de Salud, 2004), aunque focalizado en un solo distrito limeño, resalta la prevalencia de vida de Depresión Mayor en un 9.2\%, con mayor prevalencia en las mujeres (12.4\%) que en los varones (6.1\%). Además, en este mismo estudio, uno de cada tres entrevistados manifestó haber tenido síntomas depresivos en algún momento de su vida.

El Instituto Nacional de Salud Mental "Honorio Delgado-Hideyo Noguchi" (2002) señala que la prevalencia de vida de los trastornos depresivos en general es de un $19 \%$, siendo para el sexo masculino de un $14.5 \%$ y para el sexo femenino de un $23.3 \%$. Así también, la prevalencia actual de trastornos depresivos en general es de un $6.7 \%$, siendo para los varones de un $3.0 \%$ y de las mujeres de un $10.2 \%$. Es importante señalar que el género que más padece estos tipos de trastornos según estos estudios es la población femenina. Es por ello que Rondón (2006) señala que la salud mental es un problema de salud pública en nuestro país.

Los trastornos depresivos se caracterizan por la presencia de un estado de ánimo depresivo la mayor parte del día según lo indica el propio sujeto (p. e. se siente triste o vacío) o la observación realizada por otros (p. e. llanto); disminución del interés o de la capacidad para el placer en todas o casi todas las actividades; pérdida importante de peso sin hacer régimen o aumento de peso, o aumento o pérdida de apetito; insomnio o hipersomnia casi todo el día; agitación o enlentecimiento psicomotor, fatiga o pérdida de energía; sentimientos de inutilidad o de culpa excesivos o inapropiados; disminución de la capacidad para pensar o concentrarse; pensamientos recurrentes de muerte (American Psychiatric Association, 2000).

Entonces, por las consecuencias negativas que trae para la persona es que la Organización Mundial de la Salud afirma que para el año 2020, la depresión será la segunda causa de incapacidad en el mundo, lo que pone a este trastorno anímico y mental en un lugar bastante preocupante, más si se considera que las consecuencias de sufrirla se hacen palpables no solo en la vida personal y profesional, sino que también, en algunos casos, puede terminar en tragedias mayores si se le permite prosperar; e incluso esta misma entidad señala que se produce un suicidio por depresión cada 40 segundos.

Entonces, la necesidad de contar con instrumentos de evaluación estandarizados en nuestro medio para la detección de aspectos depresivos, o en su defecto, para una adecuada identificación por parte del evaluador en población clínica, se hace evidente. En nuestro medio son de uso frecuente instrumentos tales como la Escala Autoadministrada de Depresión de Zung (Zung, 1965) y el Inventario de Depresión de Beck-II (Beck, Steer, \& Brown, 2006), los cuales fueron empleados en diversos estudios en nuestro medio que utilizaron el propuesto por Zung (Perales, Chue, Padilla \& Barahona, 2011; Pereyra-Elías, et. al., 2010; Cotlear, Osada, Ceccarelli, Ruiz-Grosso \& Vega-Dienstmaier, 2009), así como el propuesto por Beck (Carranza, 2011; Acosta, Krüger-Malpartida, Huayanay-Falconí, Bravo-Puccio y Arévalo-Flores, 2010; Castillo-Vilca, Prado-Mendoza \& Vega-Dienstmaier, 2010; Rosas, Yampufé, López, Carlos \& Sotil, 2010; Lam, Contreras, Mori, Gil, Espinoza \& Córdova, 2008; Pimentel \& Bisco, 2000). Dichas escalas presentan limitaciones debido a que no presentan una diferenciación clara entre la situación actual de la persona al momento de responder al instrumento, y su manera de comportarse más frecuente. Además, la Escala Autoadministrada de Depresión de Zung (Zung, 1965) enfatiza en aspectos fisiológicos, dejando de lado el componente afectivo de la depresión. Del mismo modo, el Inventario de Depresión de Beck-II (Beck, Steer, \& Brown, 2006) plantea un formato de calificación ordinal (respuestas que van de 1 a 4), pero el contenido relacionado con la depresión de los ítems no guarda esa característica. Sumado a ello, no presentan estudios actuales que brinden evidencias de validez en población consultante o no consultante, las cuales darían un sustento más científico a su uso en diversos contextos. Por su parte, el Inventario de Depresión Estado-Rasgo (IDER) hace la diferenciación entre la situación actual de la persona, y su forma habitual de comportarse; rescata el componente afectivo de la depresión; y plantea reactivos con respuestas ordenadas según la intensidad (estado) y frecuencia (rasgo) de las conductas planteadas. 
Considerando lo mencionado líneas arriba, desde el planteamiento de Agudelo, Spielberger \& Buela-Casal (2007) se hace una diferenciación entre la frecuencia de aparición de los síntomas (rasgo) y la afectación que tiene la persona de acuerdo a dicha sintomatología (estado), permitiendo comprender como la presencia de rasgos depresivos aumenta la probabilidad de vulnerabilidad a episodios depresivos (Agudelo, 2009). Del mismo modo, esta diferenciación entre estado y rasgo, hace la prueba más sensible a cambios pequeños en su nivel de afectación, dado el carácter transitorio de éstos, y hace más probable la aplicación del instrumento en población no consultante (Agudelo, Carretero-Dios, Blanco, Pitti, Spielberger, \& Buela-Casal, 2005). Es por ello que se plantea el estudio utilizando este instrumento que, citando a Agudelo et. al., 2005), dirige específicamente su atención a las áreas constitutivas de la depresión: los trastornos afectivos.

En este sentido, Agudelo (2009) estudió las propiedades psicométricas del Inventario de Depresión Estado/Rasgo en adolescentes y universitarios de la ciudad de Bucaramanga (Colombia), en una muestra de 198 adolescentes, de edades entre 12 y 17 años; y 278 universitarios, entre 16 y 28 años de edad. Se usó además el Inventario de Depresión de Beck-revisado, el Cuestionario Básico de Depresión (Peñate, 2001), el Inventario de Ansiedad Estado-Rasgo (Spielberger, Gorsuch \& Lushene, 1970) y el Inventario de Expresión de la Ira Estado-Rasgo (Miguel Tobal, Casado, Cano \& Spielberger, 2001). Los resultados indican altos valores de confiabilidad, siendo el valor del Alfa de Cronbach para Depresión-Estado de .91 para mujeres y .86 para varones, y el Alfa de Depresión-Rasgo, de .78 y .82 para varones y mujeres, respectivamente. También se hallaron evidencias de validez convergente y discriminante, dado que se observaron correlaciones significativas entre los instrumentos ya mencionados y el Inventario de Depresión Estado/Rasgo. Con relación al análisis factorial planteado, este corrobora la estructura original del instrumento en términos de eutimia y distimia al interior de las subescalas.

Del mismo modo, Ocampo (2007) realizó un estudio correlacional del Inventario de Depresión Estado/Rasgo () en adolescentes y universitarios de la ciudad de Medellín (Colombia), usando como medida comparativa el Inventario de Depresión de Beck-revisado (Beck, Rush, Shaw \& Emery, 1979), el Cuestionario Básico de Depresión (Peñate, 2001), el Inventario de Ansiedad Estado-Rasgo (Spielberger, Gorsuch \& Lushene, 1970) y el Inventario de Expresión de la Ira Estado-Rasgo (Spielberger, 1996). Se hallaron indicadores de confiabilidad elevados, siendo el Alfa de .81 y .78 para el grupo de adolescentes y universitarios, respectivamente. Del mismo modo, existe evidencia de validez convergente y discriminante, dado que hay correlaciones significativas entre las escalas de los instrumentos utilizados. Con relación al estudio factorial realizado dentro del reporte, no hay una referencia esclarecedora acerca de la conformación de los factores luego de la rotación realizada.

En este contexto, el presente trabajo tuvo como objetivo fundamental determinar las propiedades psicométricas del Inventario de Depresión Estado Rasgo (IDER) elaborado por Spielberger, Agudelo \& Buela-Casal (2008) en cuanto a validez y confiabilidad, dado que no se han hallado estudios nacionales con este instrumento, y existe evidencia de validez y confiabilidad en otras latitudes, lo cual incentivó a la realización de este estudio, ya que así se llenará el vacío que significa la evaluación del componente afectivo de la depresión de manera específica en nuestro medio.

\section{MÉTODO}

Según León \& Montero (2002) es un estudio instrumental, destinado a la adaptación y estudio de las propiedades psicométricas de un test.

\section{Participantes}

La muestra de estudio estuvo conformada por 251 personas, de las cuales 129 fueron varones y 122 mujeres; asimismo, de esta muestra, 110 personas acudían a consulta psicológica en hospitales de Lima Metropolitana, y cuya impresión diagnóstica de acuerdo indicadores CIE-10 fue de Depresión (Media de edad: 33.65), y el grupo no consultante, el cual estuvo conformado por 141 personas, estuvo conformado por estudiantes universitarios (Media de edad: 20.44). El muestreo utilizado fue de tipo intencional. 


\section{Instrumento}

En el estudio se utilizó el Inventario de Depresión estado - Rasgo IDER. (Spielberger, Agudelo \& BuelaCasal, 2008), que consta de dos partes de diez ítems cada una, las cuales tiene como objetivo principal la evaluación del grado de afectación (estado) y la frecuencia de ocurrencia (rasgo) del componente afectivo de la depresión, considerando para ello cuatro áreas, las cuales son:

Distimia estado: grado en el que está presente en el momento de la evaluación un estado de afectividad negativa.

Eutimia estado: grado en el que está presente en el momento de la evaluación la afectividad positiva.

Distimia rasgo: frecuencia de la presencia de afectividad negativa.

Eutimia rasgo: frecuencia de la presencia de afectividad positiva.

Los ítems que reflejan Eutimia, tanto en la escala Estado como Rasgo se encuentran en escala invertida, lo cual quiere decir que a mayor puntuación, hay mayor presencia de afectividad negativa.

\section{Procedimiento}

Se realizó una adecuación lingüística de algunos ítems, la cual estuvo a cargo de jueces expertos con experiencia en psicología clínica, quienes calificaron y modificaron los ítems conservando el propósito de éstos, pero adecuando algunos a la realidad lingüística.

De manera paralela, los investigadores se pusieron en contacto con psicólogos clínicos que laboren en diversos hospitales nacionales de Lima Metropolitana, y que tengan algún día de la semana a su cargo el servicio de consulta externa, en el cual se realizan las evaluaciones.

El inventario se administró dentro de una batería de evaluación psicológica propia del hospital de referencia. El psicólogo explicó las instrucciones que figuran en el protocolo de aplicación de la prueba, y absolvió las dudas que las personas evaluadas tuvieran.

El análisis de los datos se llevó a cabo utilizando el paquete estadístico SPSS 18.0.

\section{RESULTADOS}

Confiabilidad: Se usó para este fin el coeficiente de Alpha de Cronbach, siendo los valores de .864 para Eutimia-Estado; .861 para Distimia-Estado; .793 para Eutimia-Rasgo; y .862 para Distimia-Rasgo.

Análisis de Homogeneidad del test: En esta sección se examinó el grado de asociación entre los ítems que conforman la prueba y el test (Elosua, 2003).

Tabla N. ${ }^{\circ} 1$.

Coeficientes de correlación ítem-test del Inventario de Depresión Estado-Rasgo (IDER)

\begin{tabular}{lccc}
\hline \multicolumn{2}{c}{ Eutimia Estado } & \multicolumn{2}{c}{ Distimia Estado } \\
\hline ítem & $\begin{array}{c}\text { Correlación } \\
\text { ítem-test }\end{array}$ & Ítem & $\begin{array}{c}\text { Correlación } \\
\text { ítem-test }\end{array}$ \\
Ítem 4 & .768 & Ítem 8 & .672 \\
Ítem 9 & .700 & Ítem 3 & .609 \\
Ítem 1 & .662 & Ítem 5 & .714 \\
Ítem 1 & 0.650 & Ítem 6 & .733 \\
Ítem 7 & .651 & Ítem 2 & .668 \\
\hline
\end{tabular}

Tabla N. ${ }^{\circ} 2$.

Coeficientes de correlación ítem-test del Inventario de Depresión Estado-Rasgo (IDER)

\begin{tabular}{lccc}
\hline \multicolumn{2}{c}{ Eutimia Rasgo } & \multicolumn{2}{c}{ Distimia Rasgo } \\
\hline ítem & $\begin{array}{c}\text { Correlación } \\
\text { ítem-test }\end{array}$ & ítem & $\begin{array}{c}\text { Correlación } \\
\text { ítem-test }\end{array}$ \\
Ítem 4 & .511 & Ítem 7 & .504 \\
Ítem 1 & .686 & Ítem 6 & .702 \\
Ítem 10 & .601 & Ítem 8 & .741 \\
Ítem 5 & .557 & Ítem 9 & .727 \\
Ítem 3 & .515 & Ítem 2 & .742 \\
\hline
\end{tabular}

Validez de contenido: Para este proceso, se utilizó la consulta a psicólogos expertos en el tema para determinar la pertinencia de los reactivos en lo que concierne a la evaluación del componente afectivo de la depresión. Luego de ello se utilizó la V de Aiken (Escurra, 1988) para el cálculo de la validez de contenido. 
Tabla N..$^{\circ} 3$.

Validez por criterio de jueces del Inventario de Depresión Estado-Rasgo (IDER)

\begin{tabular}{clcc}
\hline Área & Ítem & Acuerdo & V de Aiken \\
\hline Eutimia Estado & Ítem 4 & 6 & 1.00 \\
& Ítem 9 & 6 & 1.00 \\
& Ítem 1 & 6 & 1.00 \\
& Ítem 10 & 5 & .83 \\
& Ítem 7 & 5 & .83 \\
Distimia Estado & Ítem 8 & 6 & 1.00 \\
& Ítem 3 & 6 & 1.00 \\
& Ítem 5 & 6 & 1.00 \\
& Ítem 6 & 4 & .67 \\
Eutimia Rasgo & Ítem 2 & 5 & .83 \\
& Ítem 4 & 5 & .83 \\
& Ítem 1 & 6 & 1.00 \\
& Ítem 10 & 5 & .83 \\
& Ítem 5 & 5 & .83 \\
& Ítem 3 & 4 & .67 \\
Distimia Rasgo & Ítem 7 & 6 & 1.00 \\
& Ítem 6 & 5 & .83 \\
& Ítem 8 & 4 & .67 \\
& Ítem 9 & 4 & .67 \\
& Ítem 2 & 5 & .83 \\
\hline
\end{tabular}

Análisis de la dimensionalidad: Para poder entender la dimensionalidad de la escala creada se realizó un análisis factorial de ejes principales con rotación promax al cumplir con las características requeridas para dicho análisis, como son una matriz de correlaciones significativa ( $\mathrm{p}<.01)$; KMO de .854, valor considerado adecuado (Hair, Anderson, Tatham \& Black, 2005), y test de esfericidad de Bartlett significativo $(\mathrm{p}<.01)$, para Depresión-Estado. Por el lado de Depresión-Rasgo, presenta características tales como una matriz de correlaciones significativa ( $\mathrm{p}<.01)$; KMO de .866, valor considerado adecuado (Hair, Anderson, Tatham \& Black, 2005) al igual que en Depresión-Estado, y test de esfericidad de Bartlett significativo $(\mathrm{p}<.01)$. La rotación promax se justifica en ambos casos dado que fue el método de rotación elegido por los autores del test (Spielberger, Agudelo, Buela-Casal, 2008). Además, la correlación entre los factores resultantes de las dos escalas es mayor a .32 (Estado: r12= -.419; Rasgo: r12= -.497) (Tabachnick $\&$ Fidell, 2001).

En cuanto al análisis de la dimensionalidad, en ambas escalas Depresión-Estado y DepresiónRasgo, fueron extraídos dos factores que explican el $65.380 \%$ y el $60.228 \%$ respectivamente. Asimismo, los dos factores principales de cada análisis superó ampliamente el $20 \%$ de varianza explicada, lo cual es un indicador de unidimensionalidad (Carmines \& Zeller, 1979). 
Tabla N. ${ }^{\circ} 4$.

Resultados de la Rotación Promax del Análisis Factorial de la Escala Depresión Estado

\begin{tabular}{lcc}
\hline Ítems & Factor 1 & Factor 2 \\
\hline Ítem 4 & .914 & \\
Ítem 9 & .841 & \\
Ítem 1 & .711 & \\
Ítem 10 & .793 & \\
Ítem 7 & .746 & \\
Ítem 8 & & .829 \\
Ítem 3 & & .675 \\
Ítem 5 & & .894 \\
Ítem 6 & & .831 \\
Ítem 2 & & .744 \\
\hline
\end{tabular}

Validez criterial: Para ello se utilizó la diferencia de puntuaciones entre grupos de sujetos de diferentes características, consultantes y no consultantes (Prieto
Tabla N. ${ }^{\circ} 5$

Resultados de la Rotación Promax del Análisis Factorial de la Escala Depresión Rasgo

\begin{tabular}{lcc}
\hline Ítems & Factor $\mathbf{1}$ & Factor 2 \\
\hline Ítem 4 & & .683 \\
Ítem 1 & .882 \\
Ítem 10 & & .803 \\
Ítem 5 & .617 \\
Ítem 3 & & .675 \\
Ítem 7 & .710 & \\
Ítem 6 & .786 & \\
Ítem 8 & .825 & \\
Ítem 9 & .840 & \\
Ítem 2 & .842 & \\
\hline
\end{tabular}

\& Delgado, 2010), siendo la variable distintiva la presencia de síntomas depresivos, mediante la $\mathrm{T}$ de Student para muestras independientes.

Tabla N. ${ }^{\circ} 6$.

Diferencias entre sujetos consultantes y no consultantes

\begin{tabular}{lcccrc}
\hline & Categoría & Frecuencia & Media & Desv. Estándar & t(249) \\
\hline EUTIMIA & No Consultante & 141 & 11,55 & 3,224 & $-3,597^{*}$ \\
ESTADO & Consultante & 110 & 13,02 & 3,174 & \\
DISTIMIA- & No Consultante & 141 & 6,69 & 2,281 & $-6,387^{*}$ \\
ESTADO & Consultante & 110 & 9,15 & 3,791 & \\
EUTIMIA- & No Consultante & 141 & 9,91 & 2,821 & $-4,648^{*}$ \\
RASGO & Consultante & 110 & 11,69 & 3,202 & \\
DISTIMIA- & No Consultante & 141 & 7,43 & 2,306 & $-4,826^{*}$ \\
RASGO & Consultante & 110 & 9,30 & 3,804 & \\
\hline
\end{tabular}

$* \mathrm{p}<0,001$ 


\section{DISCUSIÓN}

De acuerdo con Muñiz \& Hambleton (1996), la filosofía que sustenta la adaptación de un test es que el constructo sea medido de la misma forma, pero ajustándose a las peculiaridades de la nueva población, $\mathrm{y}$ entre esos aspectos, el lenguaje usado juega un rol primordial. Asimismo, tomando las ideas de Agudelo (2009), fue pertinente la evaluación por jueces para establecer acuerdos con respecto a la forma de presentar los ítems.

Se planteó una adaptación lingüística, ya que si bien la adaptación que pretendemos realizar proviene del original español, el componente semántico de las palabras difiere según el país, ya que si aparecen fallos a este nivel, cualquier medida posterior sería inútil.

La confiabilidad, según el planteamiento de la consistencia interna, un test reúne este requisito cuando cada uno de los elementos mide la misma característica (Alarcón, 1998), es decir, hacen referencia a la consistencia del test y las relaciones entre los reactivos que la integran. Entonces, la confiabilidad se refiere al grado de varianza de las mediciones atribuibles a las fuentes de error, ya que cuando la proporción de la varianza de error es baja, el coeficiente de confiabilidad será alto (Alarcón, 1998).

Respecto al coeficiente de confiabilidad alcanzado por cada una de las escalas, de acuerdo con CampoArias \& Oviedo (2008), al estar entre .70 y .90, se puede decir que no existe una pobre correlación entre los ítems, si fuera menor de .70, y tampoco redundancia, como en los índices superiores a .90. Entonces, los coeficientes hallados son los más adecuados, ya que se encuentran entre .80 y .90 .

Se tiene en consideración, tal como lo apuntan Prieto \& Delgado (2010), la variabilidad de los índices de fiabilidad entre poblaciones, sobre todo en relación a lo que caracteriza a cada población de las muestras de personas. En consecuencia, se ha de evitar el error de considerar que la estimación de la fiabilidad procedente de un único estudio refleja la verdadera y única fiabilidad de la prueba. Se debe añadir a ello que los índices de homogeneidad están por encima de .50 en todas las escalas, lo cual da cuenta que el instrumento presenta consistencia.
Este último aspecto lo podemos corroborar al contrastar los hallazgos antedichos con los de Agudelo (2009), dado que los índices de confiabilidad en la presente investigación son mayores. Aunque por otro lado, la confiabilidad hallada por Ocampo (2007) es menor a la que se encontró aquí, no hay una clara diferenciación respecto a las escalas Eutimia Estado y Distimia Estado, ya que consideró a la Escala Estado sin diferenciar entre Eutimia y Distimia.

En cuanto a la validez de contenido, de acuerdo con Escurra (1998) se requiere un mínimo de jueces que opinen de manera favorable hacia un reactivo, siendo este número de cuatro (4) jueces de seis (6) consultados. Entonces, se puede decir que, a juicio de los expertos consultados, los ítems tienen validez de contenido, es decir, que la muestra de ítems que componen la prueba es relevante y representativa del constructo (Alarcón, 1998; Elosua, 2003).

Tanto en la Escala de Depresión Estado como Depresión Rasgo se usó el análisis factorial para explorar la dimensionalidad del test, mediante el método de ejes principales, con rotación promax, dado que los factores resultantes se encuentran correlacionados.

La estructura factorial resultante corresponde con la estructura teórica de la prueba, ya que se agrupan los cinco ítems correspondientes a Eutimia, y los últimos cinco, que conforman la Distimia, en un segundo factor, en ambas escalas, Estado y Rasgo.

Entonces, a través de un número mínimo de factores y mayor varianza explicada se trata de proponer un modelo independiente, por lo cual podemos decir que hay evidencias de validez factorial (dimensionalidad) (Elosua, 2003), ya que la covariación de un grupo de subtests o ítems define un factor, que representa una dimensión teórica subyacente a todo ellos (Alarcón, 1998).

En contraste con los hallazgos de Agudelo (2009), se puede apreciar que el análisis hecho por esta autora considerando los Componentes Principales con rotación Varimax, no le define una estructura bifactorial, por lo cual se utiliza la rotación Promax, la cual le permite tener dos factores bien diferenciados. Cabe resaltar al respecto que Ocampo (2007) no considera este 
aspecto factorial, mas se remite a presentar la varianza explicada por los dos factores hallados en la muestra de universitarios, la cual en conjunto es de 52,329\% en la Escala Estado, menor a la que hallada en este estudio (65,380\%); asimismo, la Escala Rasgo también se presenta con menor porcentaje de varianza explicada en la muestra de universitarios $(61,832 \%)$ respecto a la presente investigación $(65,380 \%)$.

Dado que la validez concurrente es adecuada para la validación de tests empleados para diagnóstico de la situación actual (Anastasi, 1974) y de acuerdo con Prieto \& Delgado (2010), las relaciones de los puntajes con otras variables externas son una importante fuente de validación, siendo una de esas fuentes la comparación de grupos de diferentes niveles respecto al constructo estudiado, es decir, la depresión. En este sentido, se compararon a personas que asistían a consulta psicológica por problemas relacionados con depresión y aquellas que no lo hacían, encontrándose diferencias estadísticamente significativas en la escala Eutimia Estado $(\mathrm{t}(249)=-3.597 ; \mathrm{p}<.001)$, Distimia Estado $(\mathrm{t}(249)=-6.387 ; \mathrm{p}<.001)$, Eutimia Rasgo $(\mathrm{t}(249)=-4.648 ; \mathrm{p}<.001)$, Distimia Rasgo $(\mathrm{t}(249)=$ -4.826; $\mathrm{p}<.001$ ) entre población consultante y no consultante. Entonces, estos resultados nos indican que existe validez criterial, ya que la variable en estudio se destaca en el grupo característico de sujetos (Alarcón, 1998), existiendo en el grupo consultante un promedio mayor en todas las escalas: en Eutimia Estado (Media: 13,02; D.E.: 3,174) y Eutimia Rasgo (Media: 11,69; D.E.: 3,202), y puntuaciones mayores en Distimia Estado (Media: 9,15; D.E.: 3,791) y Distimia Rasgo (Media: 9,30; D.E.: 3,804).

Entonces, considerando la definición de validez que hace mención Prieto \& Delgado (2010), es decir, el grado en que la evidencia empírica y la teoría apoyan la interpretación de las puntuaciones de los tests relacionada con un uso especifico, considerándola entonces como un proceso de acumulación de pruebas para apoyar la interpretación y uso de las puntuaciones, el Inventario de Depresión Estado-Rasgo es un instrumento válido y confiable para ser usado como método de valoración de los síntomas afectivos de la depresión (Agudelo, et. al., 2005).
Se recomienda, a partir de los hallazgos que se continúen estudios de recopilación de evidencias de validez, dado que así se contará, a futuro, con un instrumento sólido para la evaluación del componente afectivo de la depresión.

\section{REFERENCIAS}

Acosta, J., Krüger-Malpartida, H., Huayanay-Falconí, L., Bravo-Puccio, F. \& Arévalo-Flores, M., (2010). Depresión y síntomas depresivos en pacientes que acuden al consultorio de Dermatología de un hospital general de Lima. Revista de Neuropsiquiatría, 73(4), 126-135.

Agudelo, D. (2009). Propiedades psicométricas del Inventario de Depresión Estado/Rasgo (IDER) con adolescentes y universitarios de la ciudad de Bucaramanga. En Pensamiento psicológico, 5(12), 139-160.

Agudelo, D., Carretero-Dios, H., Blanco Picabia, A., Pitti, C., Spielberger, Ch. \& Buela- Casal, G. (2005). Evaluación del componente afectivo de la depresión: análisis factorial del ST/DEP revisado. Salud Mental, 28, 32-41.

Agudelo, D. Spielberger, C. \& Buela-Casal, G. (2007). La depresión: ¿un trastorno dimensional o categorial? Salud Mental, 30, 20-28.

American Psychiatric Association (2000). Diagnostic and statistical manual of mental disorders (DSM$I V$-TR) ( $4^{\circ}$ Edición. Texto revisado). Washington, DC.

Anastasi, A. (1974). Tests psicológicos. Madrid: Aguilar.

Alarcón, R. (1998). Nuevos modelos en la medición psicológica. Lima: Universidad de San Martín de Porres, Facultad de Ciencias de la Comunicación, Turismo y Psicología.

Beck, A., Rush, A., Shaw, B. \& Emery, G (1979). Cognitive Therapy of Depression. New York: Guilford Press.

Beck, A., Steer, R. \& Brown, G. (2006). Inventario de Depresión de Beck. Buenos Aires: Paidós. 
Campo-Arias, A. \& Oviedo, H. (2008). Propiedades psicométricas de una escala: la consistencia interna. En Revista de Salud Pública, 10(5), 831839.

Carmines, E. \& Zeller, R. (1979). Reliability and validity assessment. London: Sage.

Carranza, F. 2011; Propiedades Psicométricas del Inventario de Depresión de Beck Para Universitarios de Lima. I Congreso Nacional de Investigación, Universidad Peruana Unión, Perú. Recuperado el 20 de enero de 2012, de: http:// papiros.upeu.edu.pe/handle/123456789/103

Castillo-Vilca, M., Prado-Mendoza, C. \& VegaDienstmaier, J., 2010; Prevalencia de depresión en estudiantes del quinto año de medicina de una universidad privada de Lima. Revista de Neuropsiquiatría, 73(1), 9-14.

Cotlear, I., Osada, J., Ceccarelli, M., Ruiz-Grosso, P. \& Vega-Dienstmaier, J. (2009). Sintomatología depresiva en usuarios de cabinas públicas de internet en un distrito de Lima, Perú. Revista Peruana de Medicina Experimental y Salud Pública, 26(4), 462-65.

Elosua, P. (2003). Sobre la validez de los tests. En Psicothema, 15(2), 315-321.

Escurra, L. (1988). Cuantificación de la validez de contenido por criterio de jueces. En Revista de Psicología PUCP, 6(1-2), 103-111.

Ferrando, P. \& Anguiano, C. (2010). El análisis factorial como técnica de investigación en psicología. En Papeles del psicólogo, 31(1), 18-33.

Hair, J. F., Anderson, R. E., Tatham, R. E., \& Black, W.C. (2005). Análise Multivariada de dados. Porto Alegre: Bookman

Instituto de Salud Mental "Honorio Delgado-Hideyo Noguchi” (2002). Estudio Epidemiológico de Salud Mental 2002: Informe Resumen. Lima.

Lam, N., Contreras, H., Mori, E.; Gil, E, Espinoza, E. \& Córdova, G., 2008; Estudio de la validez interna y concurrente del inventario de depresión de Beck para atención primaria (BDI-PC) en población gestante de Lima Metropolitana, mayo - junio de 2008. Revista Peruana de Epidemiología, 13(2), $1-4$
León, I. \& Montero, O. (2002). Clasificación y descripción de las metodologías de investigación en psicología. En Journal of Clinical and Health Psychology. 2(3), 503-508.

Miguel Tobal, J., Casado, M., Cano, A. \& Spielberger, C. (2001). Manual del Inventario de Expresión de Ira Estado/Rasgo, Staxi-2. Madrid: TEA Ediciones

Muñiz, J. \& Hambleton, R. (1996). Directrices para la traducción y adaptación de los tests. En Papeles del psicólogo, 66. Recuperado el 10 de noviembre de 2011, de http://www.papelesdelpsicologo.es/ vernumeroas? $\mathrm{id}=737$

Ocampo, L. (2007). Análisis correlacional del CuestionariodeDepresiónEstado/Rasgo(ST/DEP) con una muestra de adolescentes y universitarios de la ciudad de Medellín (Colombia). Psicología desde el Caribe, 20, 28-49.

Peñate, W. (2001). Presentación de un cuestionario básico para la evaluación de los síntomas de la depresión. Análisis y Modificación de Conducta, 27, 671-869.

Perales, A. Chue, H., Padilla, A. \& Barahona, L. (2011). Estrés, ansiedad y depresión en magistrados de Lima, Perú. Revista Peruana de Medicina Experimental y Salud Pública, 28(4), 581-88.

Pereyra-Elías, R., Ocampo-Mascaró, J., Silva-Salazar, V., Vélez-Segovia, E., Da Costa-Bullón, D., ToroPolo, L. \& Vicuña-Ortega, J. (2010). Prevalencia y factores asociados con síntomas depresivos en estudiantes de ciencias de la salud de una universidad privada de Lima, Perú 2010. Revista Peruana de Medicina Experimental y Salud Pública, 27(4), 520-26.

Perú, Ministerio de Salud (2004). Lineamientos para la Acción en Salud Mental. Lima: Autores. Recuperado el 20 de noviembre de 2010, de http://www.congreso.gob.pe/comisiones/2006/ discapacidad/tematico/salud-rehabilitacion/ LineamientosSaludMental/saludmental.asp

Pimentel, M. \& Bisco, A. (2000). Niveles de ansiedad y depresión en pacientes VIH/SIDA asintomáticos y sintomáticos. Boletín de la Sociedad Peruana de Medicina Interna, 13(1). 
Prieto, G. \& Delgado, A. (2010). Fiabilidad y Validez. En Papeles del psicólogo, 31(1), 67-74.

Rondón, M. (2006). Salud mental: un problema de salud pública en el Perú. Revista Peruana de Medicina Experimental y Salud Publica, 23(4), 237-238.

Rosas, M., Yampufé, M., López, M., Carlos, G. \& Sotil, A.,2010; Niveles de depresión en estudiantes de Tecnología Médica. Anales de la Facultad de Medicina, 72(3), 183-186

Spielberger, C. (1996). Manual for the Trait-State Anger Expression Inventory. Florida: Psychologycal Assesment Resources.
Spielberger, C.D., Agudelo, D. \& Buela-Casal, G. (2008). Inventario de Depresión Estado/ Rasgo (IDER). Madrid: TEA Ediciones.

Spielberger, C., Gorsuch, R \& Lushene, R. (1970). STAI: Manual for the State-Trait Anxiety Inventory. Palo Alto: Consulting Psychologist Press.

Tabachnick, B. \& Fidell, L. (2001). Using multivariate statistics. New York: Harper \& Row.

Zung, W. (1965). A self-rating depression scale. Archive General of Psychiatry, 12, 63-70. 